\title{
Sufficient Conditions for Hamiltonicity of Graphs with Respect to Wiener Index, Hyper-Wiener Index, and Harary Index
}

\author{
Guisheng Jiang, ${ }^{1}$ Lifang Ren, ${ }^{2}$ and Guidong $Y u \mathbb{D}^{2,3}$ \\ ${ }^{1}$ School of Physics and Electronic Engineering, Anqing Normal University, Anqing 246133, China \\ ${ }^{2}$ School of Mathematics and Computation Sciences, Anqing Normal University, Anqing 246133, China \\ ${ }^{3}$ Basic Department, Hefei Preschool Education College, Hefei 230013, China \\ Correspondence should be addressed to Guidong Yu; guidongy@163.com
}

Received 30 June 2019; Revised 28 September 2019; Accepted 12 October 2019; Published 15 November 2019

Guest Editor: Jia-Bao Liu

Copyright $(2019$ Guisheng Jiang et al. This is an open access article distributed under the Creative Commons Attribution License, which permits unrestricted use, distribution, and reproduction in any medium, provided the original work is properly cited.

In this paper, with respect to the Wiener index, hyper-Wiener index, and Harary index, it gives some sufficient conditions for some graphs to be traceable, Hamiltonian, Hamilton-connected, or traceable for every vertex. Firstly, we discuss balanced bipartite graphs with $\delta(G) \geq t$, where $\delta(G)$ is the minimum degree of $G$, and gain some sufficient conditions for the graphs to be traceable or Hamiltonian, respectively. Secondly, we discuss nearly balanced bipartite graphs with $\delta(G) \geq t$ and present some sufficient conditions for the graphs to be traceable. Thirdly, we discuss graphs with $\delta(G) \geq t$ and obtain some conditions for the graphs to be traceable or Hamiltonian, respectively. Finally, we discuss $t$-connected graphs and provide some conditions for the graphs to be Hamilton-connected or traceable for every vertex, respectively.

\section{Introduction}

We consider only a simple graph. For a graph $G=(V(G), E(G))$, we use $n$ to denote $|V(G)|$ and $e(G)$ to $|E(G)|$. We write $d_{G}(x, y)$ as the minimum length of the paths between $x$ and $y$ in $G$, which is called the distance between two vertices $x$ and $y$ of $G$. Denote $\delta(G)=\min \left\{d_{G}(v), v \in V(G)\right\}$. For a bipartite graph $G=(X, Y ; E)$, if $|X|=|Y|$, it is called a balanced bipartite graph; if $|X|=|Y|+1$, it is called a nearly balanced bipartite graph. For disjoint graphs $G_{1}$ and $G_{2}$, the graph $G_{1}+G_{2}$ is called the union of $G_{1}$ and $G_{2}$, where $V\left(G_{1}+G_{2}\right)=V\left(G_{1}\right) \cup V\left(G_{2}\right)$ and $E\left(G_{1}+G_{2}\right)=E\left(G_{1}\right) \cup$ $E\left(G_{2}\right)$; the graph $G_{1} \vee G_{2}$ is called the join of $G_{1}$ and $G_{2}$, where $V\left(G_{1} \vee G_{2}\right)=V\left(G_{1}\right) \cup V\left(G_{2}\right)$ and $E\left(G_{1} \vee G_{2}\right)=$ $E\left(G_{1}+G_{2}\right) \cup\left\{x y: \forall x \in V\left(G_{1}\right), \forall y \in V\left(G_{2}\right)\right\}$. For example, $K_{n, m}=O_{n} \vee O_{m}$. The graph $\bar{G}=(V(\bar{G}), E(\bar{G}))$ means the complement of $G$, where $V(\bar{G})=V(G)$ and $E(\bar{G})=\{x y: x, y \in V(G), x y \notin E(G)\}$. The graph $\widehat{G}:=\left(X, Y ; E^{\prime}\right), E^{\prime}=\{x y: x \in X, y \in Y, x y \notin E\}$ is called the quasicomplement of bipartite $G=(X, Y ; E)$.
In [1], Wiener introduced the Wiener index, $W(G)=\sum_{v_{i}, v_{j} \in V(G)} d_{G}\left(v_{i}, v_{j}\right)$, for a connected graph $G$ in 1947.

We denote $D_{i}(G)=\sum_{v_{j} \in V(G)} d_{G}\left(v_{i}, v_{j}\right)$; then,

$$
W(G)=\frac{1}{2} \sum_{i=1}^{n} D_{i}(G)
$$

For a connected graph $G, \quad W W(G)=(1 / 2)$ $\left(\sum_{v_{i}, v_{j} \in V(G)} d_{G}\left(v_{i}, v_{j}\right)+\sum_{v_{i}, v_{j} \in V(G)} d_{G}^{2}\left(v_{i}, v_{j}\right)\right)$ is called its $h y-$ per-Wiener index, which is introduced by Klein et al. [2] in 1995.

We denote $D D_{i}(G)=\sum_{v_{j} \in V(G)} d_{G}^{2}\left(v_{i}, v_{j}\right)$; then,

$$
W W(G)=\frac{1}{4} \sum_{i=1}^{n}\left(D_{i}(G)+D D_{i}(G)\right) \text {. }
$$

In [3, 4], Plavšić et al. and Ivanciuc et al. gave the Harary index, $H(G)=\sum_{v_{i}, v_{i} \in V(G)} 1 /\left(d_{G}\left(v_{i}, v_{j}\right)\right)$ for a connected graph $G$, independently.

We denote $\widetilde{D}_{G}\left(v_{i}\right)=\sum_{v_{j} \in V(G)} 1 /\left(d_{G}\left(v_{i}, v_{j}\right)\right)$; then, 


$$
H(G)=\frac{1}{2} \sum_{i=1}^{n} \widetilde{D}_{G}\left(v_{i}\right) .
$$

For a graph $G$, if it contains a path (cycle) containing all vertices of $G$, it is traceable (Hamiltonian); if there are paths containing all vertices of $G$ between any two vertices in $G$, it is called to be Hamilton-connected; if there are paths containing all vertices of $G$ from any vertex in $G$, it is called to be traceable from every vertex.

Topological indices have attracted much attention in the literature, and they are widely applied in various fields of science and technology. Recently, some interesting results have been obtained, see [5-11]. Especially, some topological indices are used to characterize the Hamiltonian property of graphs. We refer readers to see [2, 12-21]. Among them, Hua and Ning [15] gave Hamiltonian conditions for a balanced bipartite graph with respect to the Wiener index and Harary index. Cai et al. [13], by the hyper-Wiener index, gave conditions for a balanced bipartite graph to be traceable and Hamiltonian and a $t$ connected graph to be Hamiltonian, respectively. $\mathrm{Li}$ $[18,19]$ gave Hamitonian conditions for a $t$-connected graph with respect to the Wiener index and Harary index, respectively. With the Wiener index, hyper-Wiener index, and Harary index, Yu et al. [20] gave conditions for a $t$ connected graph to be Hamilton-connected and then got conditions for it to be traceable from every vertex, as well as gave conditions for a nearly balance bipartite graph to be traceable. Especially, Liu et al. [16, 17] studied the Hamiltonian property of graphs with respect to the Wiener index and Harary index of complement of graph or quasicomplement of bipartite graph, respectively. As a continuance of these results, we also study the similar problems.

In this paper, we discuss the hamiltonicity of graph by the Wiener index, hyper-Wiener index, and Harary index for quasicomplement or complement. In Section 2, we present some notations and some lemmas needed in the following. In Sections 3 and 4, we present some conditions for a balanced bipartite graph $G$ with $\delta(G) \geq t$ to be traceable and Hamiltion, respectively. In Section 5, we give sufficient conditions for a nearly balanced bipartite graph $G$ with $\delta(G) \geq t$ to be traceable. In Sections 6 and 7, we present some conditions for a graph $G$ with $\delta(G) \geq t$ to be traceable and Hamiltonian, respectively. In Sections 8 and 9, we provide some conditions for a $t$-connected graph to be Hamilton-connected and traceable from every vertex, respectively.

\section{Preliminaries}

Especially, in the following, for bipartite graphs, we always fix their partite sets. For instance, $O_{s, t}$ and $O_{t, s}$ are seen as different bipartite graphs unless $s=t$.

A graph $G_{1} \sqcup G_{2}$ is the graph obtained from $G_{1} \vee G_{2}$ by deleting all possible edges between $X_{1}$ and $X_{2}$ and possible edges between $Y_{1}$ and $Y_{2}$, where $G_{i}\left(X_{i}, Y_{i}, E_{i}\right),(i=1,2)$ be bipartite graphs. Next, we denote some special classes of graphs:

$$
\begin{aligned}
& B_{n}^{t}=O_{t, n-t} \sqcup K_{n-t, t}\left(1 \leq t \leq \frac{n}{2}\right), \\
& C_{n}^{t}=O_{t, n-t} \sqcup K_{n-t-1, t}\left(1 \leq t \leq \frac{n}{2}\right), \\
& R_{n}^{t}=K_{t, t}+K_{n-t, n-t}\left(1 \leq t \leq \frac{n}{2}\right), \\
& Q_{n}^{t}=O_{t+1, n-t} \sqcup K_{n-t-1, t}\left(1 \leq t \leq \frac{n}{2}\right), \\
& L_{n}^{t}=K_{1} \vee\left(K_{t}+K_{n-t-1}\right),\left(1 \leq t \leq \frac{(n-1)}{2}\right), \\
& N_{n}^{t}=K_{t} \vee\left(K_{n-2 t}+t K_{1}\right),\left(1 \leq t \leq \frac{(n-1)}{2}\right), \\
& \underline{L}_{n}^{t}=K_{t+1}+K_{n-t-1},\left(1 \leq t \leq \frac{(n-1)}{2}\right), \\
& \underline{N}_{n}^{t}=K_{t} \vee\left(K_{n-2 t-1}+(t+1) K_{1}\right),\left(1 \leq t \leq \frac{(n-1)}{2}\right) .
\end{aligned}
$$

Note that $e\left(C_{n}^{t}\right)=n(n-t-1)+t^{2}$, and $C_{n}^{t}$ is not traceable.

Lemma 1. Denote $\widehat{G}$ as a quasicomplement of bipartite $G$ with $2 n$ vertices. If $\widehat{G}$ is a connected balanced bipartite graph, then

$$
W(\widehat{G}) \leq 2 n^{3}-3 n^{2}+2 n+2(n-1) e(G) .
$$

Proof. As $\widehat{G}$ is a quasicomplement,

$$
\begin{aligned}
W(\widehat{G})= & \sum_{v_{i}, v_{j} \in V(\widehat{G})} d_{\widehat{G}}\left(v_{i}, v_{j}\right), \\
= & \frac{1}{2} \sum_{j=1}^{2 n} D_{j}(\widehat{G}), \\
\leq & \frac{1}{2}\left(\sum _ { j = 1 } ^ { 2 n } \left(d_{\widehat{G}}\left(x_{j}\right)+(2 n-1)\left(n-d_{\widehat{G}}\left(x_{j}\right)\right)+2 n^{2}\right.\right. \\
& -4 n+2)), \\
= & 4 n^{3}-5 n^{2}+2 n+(1-n) \sum_{j=1}^{2 n} d_{\widehat{G}}\left(x_{j}\right), \\
= & 4 n^{3}-5 n^{2}+2 n+(1-n) \sum_{j=1}^{2 n}\left(n-d_{G}\left(x_{j}\right)\right), \\
= & 2 n^{3}-3 n^{2}+2 n+2(n-1) e(G) . \\
= & 2 n^{3}-3 n^{2}+2 n+(n-1) \sum_{j=1}^{2 n} d_{G}\left(x_{j}\right), \\
& \\
&
\end{aligned}
$$


Lemma 2. Let $\widehat{G}$ be a quasicomplement of bipartite $G$ with $2 n$ vertices. If $\widehat{G}$ is a connected balanced bipartite graph, then

$$
W W(\widehat{G}) \leq 2 n^{4}-5 n^{3}+5 n^{2}-n+\left(2 n^{2}-n-1\right) e(G) .
$$

Proof. As $\widehat{G}$ is a quasicomplement,

$$
\begin{aligned}
& W W(\widehat{G})=\frac{1}{2} \sum_{v_{i}, v_{j} \in V(\widehat{G})}\left(d_{\widehat{G}}\left(v_{i}, v_{j}\right)+d_{\widehat{G}}^{2}\left(v_{i}, v_{j}\right)\right), \\
& =\frac{1}{4} \sum_{j=1}^{2 n}\left(D_{j}(\widehat{G})+D D_{j}(\widehat{G})\right) \text {, } \\
& \leq \frac{1}{4} \sum_{j=1}^{2 n}\left(d_{\widehat{G}}\left(x_{j}\right)+(2 n-1)\left(n-d_{\widehat{G}}\left(x_{j}\right)\right)\right. \\
& \left.+2 n^{2}-4 n+2\right) \\
& +\frac{1}{4} \sum_{j=1}^{2 n}\left(d_{\widehat{G}}\left(x_{j}\right)+\left(4 n^{2}-4 n+1\right)\left(n-d_{\widehat{G}}\left(x_{j}\right)\right)\right. \\
& \left.+4(n-1)^{3}\right) \text {, } \\
& =4 n^{4}-6 n^{3}+4 n^{2}-n \\
& -\left(n^{2}-0.5 n-0.5\right) \sum_{j=1}^{2 n} d_{\widehat{G}}\left(x_{j}\right), \\
& =4 n^{4}-6 n^{3}+4 n^{2}-n \\
& -\left(n^{2}-0.5 n-0.5\right) \sum_{j=1}^{2 n}\left(n-d_{G}\left(x_{j}\right)\right), \\
& =2 n^{4}-5 n^{3}+5 n^{2}-n+\left(2 n^{2}-n-1\right) e(G) .
\end{aligned}
$$

Lemma 3. Let $\widehat{G}$ be a quasicomplement of bipartite $G$ of order $2 n$. If $\widehat{G}$ is a connected balanced bipartite graph, then

$$
H(\widehat{G}) \geq \frac{4 n^{3}-n}{4 n-2}-\frac{2 n-2}{2 n-1} e(G) .
$$

$$
\begin{aligned}
H(\widehat{G}) & =\sum_{v_{i}, v_{j} \in V(\widehat{G})} \frac{1}{d_{\widehat{G}}\left(v_{i}, v_{j}\right)}, \\
& =\frac{1}{2} \sum_{j=1}^{2 n} \widetilde{D}_{\widehat{G}}\left(v_{j}\right), \\
& \geq \frac{1}{2} \sum_{j=1}^{2 n}\left(d_{\widehat{G}}\left(x_{j}\right)+\frac{1}{2 n-1}\left(n-d_{\widehat{G}}\left(x_{j}\right)\right)+\frac{1}{2}\right), \\
& =\frac{n(4 n-1)}{2(2 n-1)}+\frac{n-1}{2 n-1} \sum_{j=1}^{2 n} d_{\widehat{G}}\left(x_{j}\right), \\
& =\frac{n(4 n-1)}{2(2 n-1)}+\frac{n-1}{2 n-1} \sum_{j=1}^{2 n}\left(n-d_{G}\left(x_{j}\right)\right), \\
& =\frac{4 n^{3}-n}{4 n-2}-\frac{n-1}{2 n-1} \sum_{j=1}^{2 n} d_{G}\left(x_{j}\right), \\
& \frac{4 n^{3}-n}{4 n-2}-\frac{2 n-2}{2 n-1} e(G) .
\end{aligned}
$$

Lemma 4. Let $\bar{G}$ be a complement of $G$ with $n$ vertices. If $\bar{G}$ is a connected graph, then

$$
W(\bar{G}) \leq \frac{1}{2} n(n-1)+(n-2) e(G) .
$$

Proof.

$$
\begin{aligned}
W(\bar{G}) & =\sum_{v_{i}, v_{j} \in V(\bar{G})} d_{\bar{G}}\left(v_{i}, v_{j}\right), \\
& =\frac{1}{2} \sum_{j=1}^{n} D_{j}(\bar{G}), \\
& \leq \frac{1}{2} \sum_{j=1}^{n}\left(d_{\bar{G}}\left(x_{j}\right)+(n-1)\left(n-1-d_{\bar{G}}\left(x_{j}\right)\right)\right), \\
& =\frac{n}{2}\left(n^{2}-2 n+1\right)+\frac{n}{2}(2-n) \sum_{j=1}^{n} d_{\bar{G}}\left(x_{j}\right),
\end{aligned}
$$

$$
\begin{aligned}
& =\frac{n}{2}\left(n^{2}-2 n+1\right)-\frac{n}{2}(n-2) \sum_{j=1}^{n}\left(n-1-d_{G}\left(x_{j}\right)\right), \\
& =\frac{n}{2}(n-1)+\frac{(n-2)}{2} \sum_{j=1}^{n} d_{G}\left(x_{j}\right), \\
& =\frac{1}{2} n(n-1)+(n-2) e(G) .
\end{aligned}
$$

Proof. As $\widehat{G}$ is a quasicomplement, 
Lemma 5. Let $\bar{G}$ be a complement of $G$ with $n$ vertices. If $\bar{G}$ is a connected graph, then

$$
W W(\bar{G}) \leq \frac{1}{2} n(n-1)+\frac{1}{2}\left(n^{2}-n-2\right) e(G) .
$$

Proof

$$
\begin{aligned}
W W(\bar{G})= & \frac{1}{2} \sum_{v_{i}, v_{j} \in V(\bar{G})}\left(d_{\bar{G}}\left(v_{i}, v_{j}\right)+d_{\bar{G}}^{2}\left(v_{i}, v_{j}\right)\right), \\
= & \frac{1}{4} \sum_{j=1}^{n}\left(D_{j}(\bar{G})+D D_{j}(\bar{G})\right), \\
\leq & \frac{1}{4} \sum_{j=1}^{n}\left(d_{\bar{G}}\left(x_{j}\right)+(n-1)\left(n-1-d_{\bar{G}}\left(x_{j}\right)\right)\right), \\
& +\frac{1}{4} \sum_{j=1}^{n}\left(d_{\bar{G}}\left(x_{j}\right)+(n-1)^{2}\left(n-1-d_{\bar{G}}\left(x_{j}\right)\right)\right) \\
= & \frac{n^{2}}{4}\left(n^{2}-2 n+1\right)+\frac{\left(2+n-n^{2}\right)}{4} \sum_{j=1}^{n} d_{\bar{G}}\left(x_{j}\right), \\
= & \frac{n^{2}}{4}\left(n^{2}-2 n+1\right)+\frac{\left(2+n-n^{2}\right)}{4} \sum_{j=1}^{n}\left(n-1-d_{G}\left(x_{j}\right)\right), \\
= & \frac{n}{2}(n-1)+\frac{\left(n^{2}-n-2\right)}{4} \sum_{j=1}^{n} d_{G}\left(x_{j}\right), \\
= & \frac{1}{2} n(n-1)+\frac{1}{2}\left(n^{2}-n-2\right) e(G) .
\end{aligned}
$$

Lemma 6. Let $\bar{G}$ be a complement of $G$ with $n$ vertices. If $\bar{G}$ is a connected graph, then

$$
H(\bar{G}) \geq \frac{1}{2}\left(n^{2}-n\right)-\frac{n-2}{n-1} e(G) .
$$

Proof

$$
\begin{aligned}
H(\bar{G}) & =\sum_{v_{i}, v_{j} \in V(\bar{G})} \frac{1}{d_{\bar{G}}\left(v_{i}, v_{j}\right)}, \\
& =\frac{1}{2} \sum_{j=1}^{n} \widetilde{D}_{\bar{G}}\left(v_{j}\right), \\
& \geq \frac{1}{2} \sum_{j=1}^{n}\left(d_{\bar{G}}\left(x_{j}\right)+\frac{1}{n-1}\left(n-1-d_{\bar{G}}\left(x_{j}\right)\right)\right), \\
& =\frac{n}{2}+\frac{n-2}{2(n-1)} \sum_{j=1}^{n} d_{\bar{G}}\left(x_{j}\right), \\
& =\frac{n^{2}-n}{2}-\frac{n-2}{2(n-1)} \sum_{j=1}^{n} d_{G}\left(x_{j}\right), \\
& =\frac{1}{2}\left(n^{2}-n\right)-\frac{n-2}{n-1} e(G) .
\end{aligned}
$$

\section{Traceable of Balanced Bipartite Graphs}

Lemma 7 (see [22]). Let $G$ be a balanced bipartite graph with $2 n$ vertices. If $\delta(G) \geq t \geq 1, n \geq 2 t+3$, and

$$
e(G)>n^{2}-n t-2 n+t^{2}+4 t+4,
$$

for some integer $t$, then $G$ is traceable or $G \subseteq Q_{n}^{t}$ or $t=1$, $G \subseteq R_{n}^{1}$.

Theorem 1. Let $\widehat{G}$ be a connected balanced bipartite graph with $2 n$ vertices. If $\delta(G) \geq t \geq 1, n \geq 2 t+3$, and

$$
W(\widehat{G})>4 n^{3}-(2 t+9) n^{2}+\left(2 t^{2}+10 t+14\right) n-2(t+2)^{2},
$$

for some integer $t$, then $G$ is traceable or $t=1$ and $G \subseteq R_{n}^{1}$.

Proof. Since $\quad W(\widehat{G})>4 n^{3}-(2 t+9) n^{2}+\left(2 t^{2}+10 t+14\right)$ $n-2(t+2)^{2}$, by Lemma 1 , we get $e(G)>n^{2}-n t-2 n+$ $t^{2}+4 t+4$. By Lemma 7 , we obtain that $G$ is traceable or $G \subseteq Q_{n}^{t}$ or $t=1, G \subseteq R_{n}^{1}$.

If $G \subseteq Q_{n}^{t}$ : because $\widehat{G}$ is connected and $\delta(G) \geq t \geq 1$, we get $e(G) \leq n^{2}-n t-n+t^{2}+t-(n-t-1)-t<n^{2}-n t-2 n+$ $t^{2}+4 t+4$. It is in contradiction with the above.

If $t=1$ : because $\widehat{G}$ is connected, $G \subseteq R_{n}^{1}$.

Theorem 2. Let $\widehat{G}$ be a connected balanced bipartite graph with $2 n$ vertices. If $\delta(G) \geq t \geq 1, n \geq 2 t+3$, and

$$
\begin{aligned}
W W(\widehat{G})> & 4 n^{4}-(2 t+10) n^{3}+\left(2 t^{2}+9 t+14\right) n^{2} \\
& -\left(t^{2}+3 t+3\right) n-(t+2)^{2},
\end{aligned}
$$

for some integer $t$, then $G$ is traceable or $t=1, G \subseteq R_{n}^{1}$.

Proof. Since $W W(\widehat{G})>4 n^{4}-(2 t+10) n^{3}+\left(2 t^{2}+9 t+14\right)$ $n^{2}-\left(t^{2}+3 t+3\right) n-(t+2)^{2}$, by Lemma $2, e(G)>n^{2}-n t-$ $2 n+t^{2}+4 t+4$. By Lemma $7, G$ is traceable or $G \subseteq Q_{n}^{t}$ or $t=1, G \subseteq R_{n}^{1}$. By the same discussion as the proof of Theorem 1 , the conclusion is established.

Theorem 3. Let $G$ be a connected balanced bipartite graph with $2 n$ vertices. If $\delta(G) \geq t \geq 1, n \geq 2 t+3$, and

$$
H(\widehat{G})<\frac{(4 t+12) n^{2}-\left(4 t^{2}+20 t-25\right) n+4 t^{2}+16 t+16}{4 n-2},
$$

for some integer $t$, then $G$ is traceable or $t=1, G \subseteq R_{n}^{1}$.

Proof. Since $H(\widehat{G})<\left((4 t+12) n^{2}-\left(4 t^{2}+20 t-25\right) n+4 t^{2}+\right.$ $16 t+16) /(4 n-2)$, by Lemma 3 , we get $e(G)>n^{2}-n t-2 n+$ $t^{2}+4 t+4$. By Lemma 7 , we obtain that $G$ is traceable or $G \subseteq Q_{n}^{t}$ or $t=1, G \subseteq R_{n}^{1}$. By the same discussion as the proof of Theorem 1, the conclusion is established. 


\section{Hamiltonian of Balanced Bipartite Graphs}

Lemma 8 (see [23]). Let $G$ be a balanced bipartite graph with $2 n$ vertices. If $\delta(G) \geq t \geq 1, n \geq 2 t+3$, and

$$
e(G)>n^{2}-n t-n+t^{2}+2 t+1,
$$

for some integer $t$, then $G$ is Hamiltonian or $G \subseteq B_{n}^{t}$.

Theorem 4. Let $\widehat{G}$ be a connected balanced bipartite graph with $2 n$ vertices. If $\delta(G) \geq t \geq 1, n \geq 2 t+3$, and

$$
W(\widehat{G})>4 n^{3}-(2 t+7) n^{2}+\left(2 t+2(t+1)^{2}+4\right) n-2(t+1)^{2},
$$

for some integer $t$, then $G$ is Hamiltonian.

Proof. Since $W(\widehat{G})>4 n^{3}-(2 t+7) n^{2}+\left(2 t+2(t+1)^{2}+4\right)$ $n-2(t+1)^{2}$, by Lemma 1 , we get $e(G)>n^{2}-n t-n+t^{2}+$ $2 t+1$. By Lemma $8, G$ is Hamiltonian or $G \subseteq B_{n}^{t}$.

If $G \subseteq B_{n}^{t}$ : because $\widehat{G}$ is connected and $\delta(G) \geq t \geq 1$, we get $e(G) \leq(n-t)^{2}+n t-(n-t)-t<n^{2}-n t-n+t^{2}+2 t+1$.

It is in contradiction with the above.

Theorem 5. Let $\widehat{G}$ be a connected balanced bipartite graph with $2 n$ vertices. If $\delta(G) \geq t \geq 1, n \geq 2 t+3$, and

$$
\begin{aligned}
W W(\widehat{G})> & 4 n^{4}-(2 t+8) n^{3}+\left(2 t^{2}+5 t+7\right) n^{2} \\
& -\left(t^{2}+t+1\right) n-(t+1)^{2}
\end{aligned}
$$

for some integer $t$, then $G$ is Hamiltonian.

Proof. Since $W W(\widehat{G})>4 n^{4}-(2 t+8) n^{3}+\left(2 t^{2}+5 t+7\right)$ $n^{2}-\left(t^{2}+t+1\right) n-(t+1)^{2}$, by Lemma 2 , we get $e(G)>n^{2}-$ $n t-n+t^{2}+2 t+1$. By Lemma $8, G$ is Hamiltonian or $G \subseteq B_{n}^{t}$. By the same discussion as the proof of Theorem 4, the conclusion is established.

Theorem 6. Let $\widehat{G}$ be a connected balanced bipartite graph of with $2 n$ vertices. If $\delta(G) \geq t \geq 1, n \geq 2 t+3$, and

$$
H(\widehat{G})<\frac{(4 t+8) n^{2}-\left(4 t^{2}+12 t+9\right) n+4 t^{2}+8 t+4}{4 n-2},
$$

for some integer $t$, then $G$ is Hamiltonian.

Proof. Since $H(\widehat{G})<\left(\left((4 t+8) n^{2}-\left(4 t^{2}+12 t+9\right) n+4 t^{2}+\right.\right.$ $8 t+4) /(4 n-2))$, by Lemma 3, $e(G)>n^{2}-n t-n+t^{2}+$ $2 t+1$. By Lemma $8, G$ is Hamiltonian or $G \subseteq B_{n}^{t}$. By the same discussion as the proof of Theorem 4, the conclusion is established.

\section{Traceable of Nearly Balanced Bipartite Graphs}

Lemma 9 (Yu, Fang, and Fan [24]). Let $G$ be a nearly balanced bipartite graph with $2 n-1$ vertices. If $\delta(G) \geq t \geq 1$, $n \geq 2 t+1$, and

$$
e(G)>n^{2}-n t-2 n+t^{2}+2 t+1,
$$

for some integer $t$, then $G$ is traceable or $G \subseteq C_{n}^{t}$.

Theorem 7. Let $\widehat{G}$ be a connected nearly balanced bipartite graph with $2 n-1$ vertices. If $n \geq 2 t+1, \delta(G) \geq t \geq 1$, and

$$
\begin{aligned}
W(\widehat{G})> & 4 n^{3}-(2 t+14) n^{2}+\left(4 t+2(t+1)^{2}+16\right) n \\
& -4(t+1)^{2}-4,
\end{aligned}
$$

for some integer $t$, then $G$ is traceable.

Proof. Let $G=G(X, Y ; E)$, where $X=\left\{x_{1}, x_{2}, \ldots, x_{n}\right\}$ and $Y=\left\{y_{1}, y_{2}, \ldots, y_{n-1}\right\}$, then

$$
\begin{aligned}
& W(\widehat{G})=\sum_{v_{i}, v_{j} \in V(\widehat{G})} d_{\widehat{G}}\left(v_{i}, v_{j}\right) \\
& =\frac{1}{2} \sum_{j=1}^{2 n-1} D_{j}(\widehat{G}) \\
& \leq \frac{1}{2}\left(\sum _ { j = 1 } ^ { n } \left(d_{\widehat{G}}\left(x_{j}\right)+(2 n-3)\left(n-1-d_{\widehat{G}}\left(x_{j}\right)\right)\right.\right. \\
& \left.\left.+2\left(n^{2}-2 n+1\right)\right)\right) \\
& +\frac{1}{2}\left(\sum _ { j = 1 } ^ { n - 1 } \left(d_{\widehat{G}}\left(y_{j}\right)+(2 n-3)\left(n-d_{\widehat{G}}\left(y_{j}\right)\right)\right.\right. \\
& \left.\left.+2\left(n^{2}-4 n+4\right)\right)\right) \\
& =4 n^{3}-12 n^{2}+12 n-4-(n-2) \\
& \cdot\left(\sum_{j=1}^{n} d_{\widehat{G}}\left(x_{j}\right)+\sum_{j=1}^{n-1} d_{\widehat{G}}\left(y_{j}\right)\right) \\
& =2 n^{3}-6 n^{2}+8 n-4+(n-2) \\
& \cdot\left(\sum_{j=1}^{n} d_{G}\left(x_{j}\right)+\sum_{j=1}^{n-1} d_{G}\left(y_{j}\right)\right) \\
& =2 n^{3}-6 n^{2}+8 n-4+2(n-2) e(G),
\end{aligned}
$$

where $d_{\widehat{G}}\left(x_{j}\right)=n-1-d_{G}\left(x_{j}\right)$ and $d_{\widehat{G}}\left(y_{j}\right)=n-d_{G}\left(y_{j}\right)$. Because $W(\widehat{G})>4 n^{3}-(2 t+14) n^{2}+\left(4 t+2(t+1)^{2}+16\right) n-$ $4(t+1)^{2}-4$, we get $e(G)>n^{2}-n t-2 n+t^{2}+2 t+1$. By Lemma 9, we get $G$ is traceable or $G \subseteq C_{n}^{t}$. 
If $G \subseteq C_{n}^{t}$ : because $\widehat{G}$ is connected and $\delta(G) \geq t \geq 1$, we get $e(G) \leq t^{2}+n(n-t-1)-(n-t-1)-t<n^{2}-n t-2 n+t^{2}+$ $2 t+1$. It is in contradiction with the above.

Theorem 8. Let $\widehat{G}$ be a connected nearly balanced bipartite graph with $2 n-1$ vertices. If $n \geq 2 t+1, \delta(G) \geq t \geq 1$, and

$$
\begin{aligned}
W W(\widehat{G})> & 4 n^{4}-(2 t+18) n^{3}+\left(2 t^{2}+9 t+\frac{65}{2}\right) n^{2} \\
& -\left(5 t^{2}+12 t+\frac{53}{2}\right) n+2 t^{2}+4 t+8,
\end{aligned}
$$

for some integer $t$, then $G$ is traceable.

Proof. Let $G=G(X, Y: E)$, where $X=\left\{x_{1}, x_{2}, \ldots, x_{n}\right\}$ and $Y=\left\{y_{1}, y_{2}, \ldots, y_{n-1}\right\}$, then

$$
\begin{aligned}
& W W(\widehat{G})=\frac{1}{2} \sum_{v_{i}, v_{j} \in V(\widehat{G})}\left(d_{\widehat{G}}\left(v_{i}, v_{j}\right)+d_{\widehat{G}}^{2}\left(v_{i}, v_{j}\right)\right) \\
& =\frac{1}{4} \sum_{j=1}^{2 n-1}\left(D_{j}(\widehat{G})+D D_{j}(\widehat{G})\right) \\
& \leq \frac{1}{4} \sum_{j=1}^{n}\left(d_{\widehat{G}}\left(x_{j}\right)+(2 n-3)\left(n-1-d_{\widehat{G}}\left(x_{j}\right)\right)\right. \\
& \left.+2\left(n^{2}-2 n+1\right)\right) \\
& +\frac{1}{4} \sum_{j=1}^{n}\left(d_{\widehat{G}}\left(x_{j}\right)+(2 n-3)^{2}\left(n-1-d_{\widehat{G}}\left(x_{j}\right)\right)\right. \\
& \left.+4(n-1)^{2}(n-1)\right) \\
& +\frac{1}{4} \sum_{j=1}^{n-1}\left(d_{\widehat{G}}\left(y_{j}\right)+(2 n-3)\left(n-d_{\widehat{G}}\left(y_{j}\right)\right)\right. \\
& +(2 n-4)(n-2)) \\
& +\frac{1}{4} \sum_{j=1}^{n-1}\left(d_{\widehat{G}}\left(y_{j}\right)+(2 n-3)^{2}\left(n-d_{\widehat{G}}\left(y_{j}\right)\right)\right. \\
& \left.+(2 n-4)^{2}(n-2)\right) \\
& =4 n^{4}-16 n^{3}+\frac{51}{2} n^{2}-\frac{39}{2} n+6+\frac{1}{4}\left(-4 n^{2}+10 n-4\right) \\
& \cdot\left(\sum_{j=1}^{n} d_{\widehat{G}}\left(x_{j}\right)+\sum_{j=1}^{n-1} d_{\widehat{G}}\left(y_{j}\right)\right) \\
& =2 n^{4}-9 n^{3}+\frac{37}{2} n^{2}-\frac{35}{2} n+6+\frac{1}{4}\left(4 n^{2}-10 n+4\right) \\
& \cdot\left(\sum_{j=1}^{n} d_{G}\left(x_{j}\right)+\sum_{j=1}^{n-1} d_{G}\left(y_{j}\right)\right) \\
& =2 n^{4}-9 n^{3}+\frac{37}{2} n^{2}-\frac{35}{2} n+6+\left(2 n^{2}-5 n+2\right) e(G) \text {, }
\end{aligned}
$$

where $d_{\widehat{G}}\left(x_{j}\right)=n-1-d_{G}\left(x_{j}\right)$ and $d_{\widehat{G}}\left(y_{j}\right)=n-d_{G}\left(y_{j}\right)$. Because $W W(\widehat{G})>4 n^{4}-(2 t+18) n^{3}+\left(2 t^{2}+9 t+(65 / 2)\right)$ $n^{2}-\left(5 t^{2}+12 t+(53 / 2)\right) n+2 t^{2}+4 t+8$, we get $e(G)>n^{2}-$ $n t-2 n+t^{2}+2 t+1$. By Lemma 9 , we obtain that $G$ is traceable or $G \subseteq C_{n}^{t}$. By the same discussion as the proof of Theorem 7, the conclusion is established.

Theorem 9. Let $\widehat{G}$ be a connected nearly balanced bipartite graph with $2 n-1$ vertices. If $n \geq 2 t+1, \delta(G) \geq t \geq 1$, and

$$
H(\widehat{G}) \leq \frac{(4 t+8) n^{2}-\left(4 t^{2}+16 t+17\right) n+8 t^{2}+16 t+8}{4 n-6},
$$

for some integer $t$, then $G$ is traceable or $G \subseteq C_{n}^{t}(t \leq 6)$.

Proof. Let $G=G(X, Y: E)$, where $X=\left\{x_{1}, x_{2}, \ldots, x_{n}\right\}$ and $Y=\left\{y_{1}, y_{2}, \ldots, y_{n-1}\right\}$, then

$$
\begin{aligned}
H(\widehat{G})= & \sum_{v_{i}, v_{j} \in V(G)} \frac{1}{d_{\widehat{G}}\left(v_{i}, v_{j}\right)} \\
= & \frac{1}{2} \sum_{j=1}^{n} \widetilde{D}_{\widehat{G}}\left(v_{j}\right) \\
\geq & \frac{1}{2} \sum_{j=1}^{n}\left(d_{\widehat{G}}\left(x_{j}\right)+\frac{1}{2 n-3}\left(n-1-d_{\widehat{G}}\left(x_{j}\right)\right)+\frac{1}{2}\right) \\
& +\frac{1}{2} \sum_{j=1}^{n-1}\left(d_{\widehat{G}}\left(y_{j}\right)+\frac{1}{2 n-3}\left(n-d_{\widehat{G}}\left(y_{j}\right)\right)+\frac{1}{2}\right) \\
= & \frac{4 n^{2}-5 n}{4 n-6}+\frac{n-2}{2 n-3}\left(\sum_{j=1}^{n} d_{\widehat{G}}\left(x_{j}\right)+\sum_{j=1}^{n-1} d_{\widehat{G}}\left(y_{j}\right)\right) \\
= & \frac{4 n^{3}-8 n^{2}+3 n}{4 n-6}-\frac{n-2}{2 n-3}\left(\sum_{j=1}^{n} d_{G}\left(x_{j}\right)+\sum_{j=1}^{n-1} d_{G}\left(y_{j}\right)\right) \\
= & \frac{4 n^{3}-8 n^{2}+3 n}{4 n-6}-\frac{2(n-2)}{2 n-3} e(G),
\end{aligned}
$$

where $d_{\widehat{G}}\left(x_{j}\right)=n-1-d_{G}\left(x_{j}\right)$ and $d_{\widehat{G}}\left(y_{j}\right)=n-d_{G}\left(y_{j}\right)$. Because

$$
H(\widehat{G}) \leq \frac{(4 t+8) n^{2}-\left(4 t^{2}+16 t+17\right) n+8 t^{2}+16 t+8}{4 n-6},
$$

we get $e(G)>n^{2}-n t-2 n+t^{2}+2 t+1$. By Lemma 9, we obtain that $G$ is traceable or $G \subseteq C_{n}^{t}$. Note that $t \geq 7$ and $H\left(C_{n}^{t}\right)=(1 / 4)\left(n^{2}+2 t n-n-2 t^{2}\right)>\left((4 t+8) n^{3}-\left(4 t^{2}+24\right.\right.$ $\left.t+33) n^{2}+\left(16 t^{2}+48 t+44\right) n-16 t^{2}-32 t-19\right) /\left(4 n^{2}-14 n\right.$ $+12)$. If $G \subseteq C_{n}^{t}$, then $H(\widehat{G})>H\left(\widehat{C_{n}^{t}}\right)$. The conclusion is established. 


\section{Traceable of Graphs}

Lemma 10 (see [23]). Let $G$ be a graph with $n(\geq 6 t+10)$ vertices, where $t$ is an integer. If $\delta(G) \geq t$ and $e(G)>$ $\left(\begin{array}{c}n-t-2 \\ 2\end{array}\right)+t^{2}+3 t+2$, then $G$ is traceable unless $G \subseteq \underline{L}_{n}^{t}$ or $\underline{N}_{n}^{t}$.

Theorem 10. Let $\bar{G}$ be a connected graph with $n(\geq 6 t+10)$ vertices, where $t$ is an integer. If $\delta(G) \geq t$ and

$$
\begin{aligned}
W(\bar{G})> & \frac{1}{2}\left(n^{3}-(2 t+6) n^{2}+\left(3 t^{2}+15 t+19\right) n-6 t^{2}\right. \\
& -22 t-20),
\end{aligned}
$$

then $G$ is traceable.

Proof. Since $W(\bar{G})>(1 / 2)\left(n^{3}-(2 t+6) n^{2}+\left(3 t^{2}+15 t+\right.\right.$ 19) $n-6 t^{2}-22 t-20$ ), by Lemma 4 , we get $e(G)>$ $\left(\begin{array}{c}n-t-2 \\ 2\end{array}\right)+t^{2}+3 t+2$. By Lemma 10 , we obtain that $G$ is traceable unless $G \subseteq \underline{L}_{n}^{t}$ or $\underline{N}_{n}^{t}$.

If $G \subseteq \underline{L}_{n}^{t}$ : note that $W\left(\underline{\underline{L}}_{n}^{t}\right)=n^{2}-2 n-t n+t^{2}+2 t+1$. Then, if $G \subseteq \underline{L}_{n}^{t}$, we have $W(\bar{G}) \leq W\left(\underline{L}_{n}^{t}\right)<(1 / 2)\left(n^{3}-(2 t+\right.$ 6) $\left.n^{2}+\left(3 t^{2}+15 t+19\right) n-6 t^{2}-22 t-20\right)$, a contradiction.

If $G \subseteq \underline{N}_{n}^{t}$ : note that $W\left(\overline{N_{n}^{t}}\right)=(1 / 2)\left(2 n^{2}-4 n-6 t n+\right.$ $\left.5 t^{2}+7 t+2\right)$. Then, if $G \subseteq \underline{N}_{n}^{t}$, we have $W(\bar{G}) \leq W\left(\bar{N}_{n}^{t}\right)<$ $(1 / 2)\left(n^{3}-(2 t+6) n^{2}+\left(3 t^{2}+15 t+19\right) n-6 t^{2}-22 t-20\right)$, a contradiction.

Theorem 11. Let $\bar{G}$ be a connected graph with $n(\geq 6 t+10)$ vertices, where $t$ is an integer. If $\delta(G) \geq t$ and

$$
\begin{gathered}
W W(\bar{G})>\frac{1}{4} n^{4}-\left(\frac{1}{2} t+\frac{3}{2}\right) n^{3}+\left(\frac{3}{4} t^{2}+\frac{13}{4} t+\frac{15}{4}\right) n^{2} \\
-\left(\frac{3}{4} t^{2}+\frac{7}{4} t+\frac{1}{2}\right) n-\frac{3}{2} t^{2}-\frac{11}{2} t-5,
\end{gathered}
$$

then $G$ is traceable.

Proof. Since $W W(\bar{G})>(1 / 4) n^{4}-((1 / 2) t+(3 / 2)) n^{3}+((3 /$ $\left.4) t^{2}+(13 / 4) t+(15 / 4)\right) n^{2}-\left((3 / 4) t^{2}+(7 / 4) t+(1 / 2)\right) n-(3 /$ $2) t^{2}-(11 / 2) t-5$, by Lemma 5 , we get $e(G)>$ $\left(\begin{array}{c}n-t-2 \\ 2\end{array}\right)+t^{2}+3 t+2$. By Lemma 10 , we obtain that $G$ is traceable or $G \subseteq \underline{L}_{n}^{t}$ or $\underline{N}_{n}^{t}$.

If $G \subseteq \underline{L}_{n}^{t}$ : note that $W W\left(\overline{\underline{L}_{n}^{t}}\right)=(1 / 2)\left(3 n^{2}-7 n-4 t n+\right.$ $\left.4 t^{2}+8 t+4\right)$. Then, if $G \subseteq \underline{L}_{n}^{t}$, we have $W W(\bar{G}) \leq W W\left(\overline{L_{n}^{t}}\right)$ $<(1 / 4) n^{4}-((1 / 2) t+(3 / 2)) n^{3}+\left((3 / 4) t^{2}+(13 / 4) t+(15 /\right.$ 4) $) n^{2}-\left((3 / 4) t^{2}+(7 / 4) t+(1 / 2)\right) n-(3 / 2) t^{2}-(11 / 2) t-5$, a contradiction.

If $G \subseteq \underline{N}_{n}^{t}$ : note that $W W\left(\overline{N_{n}^{t}}\right)=3 n^{2}-7 n-10 t n+9 t^{2}+$ $13 t+4$. Then, if $G \subseteq \underline{N}_{n}^{t}$, we have $W W(\bar{G}) \leq W W\left(\bar{N}_{n}^{t}\right)<(1 /$ $4) n^{4}-((1 / 2) t+(3 / 2)) n^{3}+\left((3 / 4) t^{2}+(13 / 4) t+(15 / 4)\right) n^{2}-$ $\left((3 / 4) t^{2}+(7 / 4) t+(1 / 2)\right) n-(3 / 2) t^{2}-(11 / 2) t-5$, a contradiction.

Theorem 12. Let $\bar{G}$ be a connected graph with $n(\geq 6 t+10)$ vertices, where $t$ is an integer. If $\delta(G) \geq t$ and

$$
H(\bar{G})<\frac{(2 t+4) n^{2}-\left(3 t^{2}+15 t+18\right) n+6 t^{2}+22 t+20}{2 n-2},
$$

then $G$ is traceable.

Proof. Since $H(\bar{G})<\left(\left((2 t+4) n^{2}-\left(3 t^{2}+15 t+18\right) n+6 t^{2}+\right.\right.$ $22 t+20) /(2 n-2))$, by Lemma 6 , we get $e(G)>\left(\begin{array}{c}n-t-2 \\ 2\end{array}\right)+t^{2}+3 t+2$. By Lemma 10, we obtain that $G$ is traceable unless $G \underline{\subseteq} \underline{L}_{n}^{t}$ or $\underline{N}_{n}^{t}$.

If $G \subseteq \underline{L}_{n}^{t}$ : note that $H\left(\underline{L}_{n}^{t}\right)=(1 / 4)\left(n^{2}+n+2 t n-2 t^{2}-\right.$ 2). Then, if $G \subseteq \underline{L}_{n}^{t}$, we have $H(\bar{G}) \geq H\left(\overline{L_{n}^{t}}\right)>\left(\left((2 t+4) n^{2}-\right.\right.$ $\left.\left.\left(3 t^{2}+15 t+18\right) n+6 t^{2}+22 t+20\right) /(2 n-2)\right)$, a contradiction. If $G \subseteq \underline{N}_{n}{ }^{t}$ : note that $H\left(\bar{N}_{n}^{t}\right)=(1 / 4)\left(n^{2}+n+2 t n-2 t^{2}-\right.$ $6 t-2)$. Then, if $G \subseteq \underline{N}_{n}^{t}$, we have $H(\bar{G}) \geq H\left(\bar{N}_{n}^{t}\right)>(((2 t+$ 4) $\left.\left.n^{2}-\left(3 t^{2}+15 t+18\right) n+6 t^{2}+22 t+20\right) /(2 n-2)\right)$, a contradiction.

\section{Hamiltonian of Graphs}

Lemma 11 (see [23]). Let $G$ be a graph with $n(\geq 6 t+5)$ vertices, where $t$ is an integer. If $\delta(G) \geq t$ and

$$
e(G)>\left(\begin{array}{c}
n-t-1 \\
2
\end{array}\right)+t^{2}+2 t+1,
$$

then $G$ is Hamiltonian or $G \subseteq L_{n}^{t}$ or $N_{n}^{t}$.

Theorem 13. Let $\bar{G}$ be a connected graph with $n(\geq 6 t+5)$ vertices, where $t$ is an integer. If $\delta(G) \geq t$ and

$$
\begin{aligned}
W(\bar{G})> & \frac{1}{2}\left(n^{3}-(2 t+4) n^{2}+\left(3 t^{2}+11 t+19\right) n\right. \\
& \left.-6 t^{2}-14 t-8\right),
\end{aligned}
$$

then $G$ is Hamiltonian.

Proof. Since $W(\bar{G})>(1 / 2)\left(n^{3}-(2 t+4) n^{2}+\left(3 t^{2}+11 t+\right.\right.$ 19) $\left.n-6 t^{2}-14 t-8\right)$, by Lemma 4 , we get $e(G)>$ $\left(\begin{array}{c}n-t-1 \\ 2\end{array}\right)+t^{2}+2 t+1$. By Lemma 11 , we obtain that $G$ is Hamiltonian or $G \subseteq L_{n}^{t}$ or $N_{n}^{t}$.

If $G \subseteq L_{n}^{t}$ : note that $W\left(\overline{L_{n}^{t}}\right)=n^{2}-t n-3 n+t^{2}+t+2$. Then, if $G \subseteq L_{n}^{t}$, we have $W(\bar{G}) \leq W\left(\overline{L_{n}^{t}}\right)<(1 / 2)\left(n^{3}-(2 t+\right.$ 4) $\left.n^{2}+\left(3 t^{2}+11 t+19\right) n-6 t^{2}-14 t-8\right)$, a contradiction.

If $G \subseteq N_{n}^{t}$ : note that $W\left(\overline{N_{n}^{t}}\right)=n^{2}-3 t n-n+(5 / 2) t^{2}+$ $(3 / 2) t$. Then, if $G \subseteq N_{n}^{t}$, we have $W(\bar{G}) \leq W\left(N_{n}^{t}\right)<$ 
$(1 / 2)\left(n^{3}-(2 t+4) n^{2}+\left(3 t^{2}+11 t+19\right) n-6 t^{2}-14 t-8\right)$, a contradiction.

Theorem 14. Let $\delta(G) \geq t$ and $\bar{G}$ be a connected graph with $n(\geq 6 t+5)$ vertices for some integers $t$ satisfying the following condition:

$$
\begin{aligned}
W W(\bar{G})> & \frac{1}{4} n^{4}-\left(\frac{1}{2} t+1\right) n^{3}+\left(\frac{3}{4} t^{2}+\frac{9}{4} t+\frac{3}{2}\right) n^{2} \\
& -\left(\frac{3}{4} t^{2}+\frac{3}{4} t+\frac{1}{4}\right) n-\left(\frac{3}{2} t^{2}+\frac{7}{2} t+2\right) .
\end{aligned}
$$

Therefore, $G$ is Hamiltonian.

Proof. Since $W W(\bar{G})>(1 / 4) n^{4}-((1 / 2) t+1) n^{3}+\left((3 / 4) t^{2}\right.$ $+(9 / 4) t+(3 / 2)) n^{2}-\left((3 / 4) t^{2}+(3 / 4) t+(1 / 4)\right) n-\left((3 / 2) t^{2}+\right.$ $(7 / 2) t+2)$, by Lemma 5 , we get $e(G)>$ $\left(\begin{array}{c}n-t-1 \\ 2\end{array}\right)+t^{2}+2 t+1$. By Lemma 11 , we obtain that $G$ is Hamiltonian or $G \subseteq L_{n}^{t}$ or $N_{n}^{t}$.

If $G \subseteq L_{n}^{t}$ : note that $W W\left(\overline{L_{n}^{t}}\right)=3 n^{2}-4 t n-9 n+$ $4 t^{2}+4 t+6$. Then, if $G \subseteq L_{n}^{t}$, we have $W W(\bar{G})$ $\leq W W\left(\overline{L_{n}^{t}}\right)<(1 / 4) n^{4}-((1 / 2) t+1) n^{3}+\left((3 / 4) t^{2}+(9 / 4) t+\right.$ $(3 / 2)) n^{2}-\left((3 / 4) t^{2}+\quad(3 / 4) t+(1 / 4)\right) n-\left((3 / 2) t^{2}+(7 / 2)\right.$ $t+2)$, a contradiction.

If $G \subseteq N_{n}^{t}$ : note that $W W\left(\overline{N_{n}^{t}}\right)=3 n^{2}-10 t n-3 n+9 t^{2}$ $+5 t$. Then, if $G \subseteq N_{n}^{t}$, we have $W W(\bar{G}) \leq W W\left(\overline{N_{n}^{t}}\right)<$ $(1 / 4) n^{4}-((1 / 2) t+1) n^{3}+\left((3 / 4) t^{2}+(9 / 4) t+(3 / 2)\right) n^{2}-((3 /$ $\left.4) t^{2}+(3 / 4) t+(1 / 4)\right) n-\left((3 / 2) t^{2}+(7 / 2) t+2\right)$, a contradiction.

Theorem 15. Let $\bar{G}$ be a connected graph with $n(\geq 6 t+5)$ vertices for some integer $t$. If $\delta(G) \geq t$ and $H(\bar{G})<(((2 t$ $\left.\left.+2) n^{2}-\left(3 t^{2}+11 t+8\right) n+6 t^{2}+14 t+8\right) /(2 n-2)\right)$, then $G$ is Hamiltonian.

Proof. Since $H(\bar{G}) \leq\left(\left((2 t+2) n^{2}-\left(3 t^{2}+11 t+8\right) n+6 t^{2}+\right.\right.$ $14 t+8) /(2 n-2))$, by Lemma 6 , we get $e(G)>$ $\left(\begin{array}{c}n-t-1 \\ 2\end{array}\right)+t^{2}+2 t+1$. By Lemma 11 , we obtain that $G$ is Hamiltonian unless $G \subseteq L_{n}^{t}$ or $N_{n}^{t}$.

If $G \subseteq L_{n}^{t}$ : note that $H\left(\overline{L_{n}^{t}}\right)=(1 / 4)\left(n^{2}-3 n+2 t n-\right.$ $\left.2 t^{2}-2 t+2\right)$. Then, if $G \subseteq L_{n}^{t}$, we have $H(\bar{G}) \geq H\left(\overline{L_{n}^{t}}\right)>\left(\left((2 t+2) n^{2}-\left(3 t^{2}+11 t+8\right) n+6 t^{2}+14 t\right.\right.$ $+8) /(2 n-2))$, a contradiction.

If $G \subseteq N_{n}^{t}$ : note that $H\left(\overline{N_{n}^{t}}\right)=(1 / 4)\left(n^{2}-n-3 t^{2}+t\right)$. Then, if $G \subseteq N_{n}^{t}$, we have $H(\bar{G}) \geq H\left(\overline{N_{n}^{t}}\right)>$ $\left(\left((2 t+2) n^{2}-\left(3 t^{2}+11 t+8\right) n+6 t^{2}+14 t+8\right) /(2 n-2)\right), \quad$ a contradiction.

\section{Hamilton-Connected of Graphs}

Lemma 12 (see [20]). Let $G$ be a t-connected graph with $n$ vertices, where $t \geq 2$. If $e(G)>((n(n-1)-t(n-t-1)) / 2)$, then $G$ is Hamilton-connected.

By Lemmas 4, 5, 6, and 12 and by direct computations, we get Theorems 16,17, and 18, respectively.
Theorem 16. Let $G$ be a $t$-connected graph with $n$ vertices and $\bar{G}$ be a connected graph, where $t \geq 2$. If

$$
W(\bar{G})>\frac{1}{2} n^{3}-\frac{1}{2}(t+2) n^{2}+\frac{1}{2}\left(t^{2}+3 t+1\right) n-t^{2}-t,
$$

then $G$ is Hamilton-connected.

Theorem 17. Let $G$ be a $t$-connected graph with $n$ vertices and $\bar{G}$ be a connected graph, where $t \geq 2$. If

$$
\begin{aligned}
W W(\bar{G})> & \frac{1}{4} n^{4}-\frac{1}{4}(t+2) n^{3}+\frac{1}{4}\left(t^{2}+2 t+1\right) n^{2} \\
& -\frac{1}{4}\left(t^{2}-t\right) n-\frac{1}{2} t^{2}-\frac{1}{2} t,
\end{aligned}
$$

then $G$ is Hamilton-connected.

Theorem 18. Let $G$ be a $t$-connected graph with $n$ vertices and $\bar{G}$ be a connected graph, where $t \geq 2$. If

$$
H(\bar{G})<\frac{(t+1) n^{2}-\left(t^{2}+3 t+1\right) n+2 t^{2}+2 t}{2(n-1)},
$$

then $G$ is Hamilton-connected.

\section{Traceable from Every Vertex of Graphs}

Lemma 13 (see [20]). Let $G$ be a t-connected graph with $n$ vertices, where $t \geq 2$. If $e(G)>((n(n-1)-t(n-t)) / 2)$, then $G$ is traceable from every vertex.

By Lemmas 4, 5, 6, and 13 and by direct computation, we get Theorems 19, 20, and 21, respectively.

Theorem 19. Let $G$ be a $t$-connected graph with $n$ vertices and $\bar{G}$ be a connected graph, where $t \geq 2$. If

$$
W(\bar{G})>\frac{1}{2} n^{3}-\left(\frac{1}{2} t+1\right) n^{2}+\frac{1}{2}\left(t^{2}+2 t+1\right) n-t^{2}
$$

then $G$ is traceable from every vertex.

Theorem 20. Let $G$ be a $t$-connected graph with $n$ vertices and $\bar{G}$ be a connected graph, where $t \geq 2$. If

$$
\begin{aligned}
W W(\bar{G})> & \frac{1}{4} n^{4}-\frac{1}{4}(t+2) n^{3}+\frac{1}{4}\left(t^{2}+t+1\right) n^{2} \\
& -\frac{1}{4}\left(t^{2}-2 t\right) n-\frac{1}{2} t^{2}
\end{aligned}
$$

then $G$ is traceable from every vertex.

Theorem 21. Let $G$ be a $t$-connected graph with $n$ vertices and $\bar{G}$ be a connected graph, where $t \geq 2$. If

$$
H(\bar{G})<\frac{(t+1) n^{2}+\left(-t^{2}-2 t-1\right) n+2 t^{2}}{2 n-2},
$$

then $G$ is traceable from every vertex. 


\section{Data Availability}

The data used to support the findings of this study are included within the article.

\section{Conflicts of Interest}

The authors declare that there are no conflicts of interest regarding the publication of this paper.

\section{Acknowledgments}

This work was jointly supported by the National Natural Science Foundation of China (grant no. 11871077), the Natural Science Foundation of Anhui Province (grant no. 1808085MA04), and the Natural Science Foundation of Department of Education of Anhui Province (grant no. KJ2017A362).

\section{References}

[1] H. Wiener, "Structural determination of paraffin boiling points," Journal of the American Chemical Society, vol. 69, no. 1, pp. 17-20, 1947.

[2] D. J. Klein, I. Lukovits, and I. Gutman, "On the definition of the hyper-wiener index for cycle-containing structures," Journal of Chemical Information and Modeling, vol. 35, no. 1, pp. 50-52, 1995.

[3] O. Ivanciuc, T.-S. Balaban, and A. T. Balaban, "Design of topological indices. Part 4. Reciprocal distance matrix, related local vertex invariants and topological indices," Journal of Mathematical Chemistry, vol. 12, no. 1, pp. 309-318, 1993.

[4] D. Plavšić, S. Nikolić, N. Trinajstić, and Z. Mihalić, "On the Harary index for the characterization of chemical graphs," Journal of Mathematical Chemistry, vol. 12, no. 1, pp. 235-250, 1993.

[5] Y. Gao, E. Zhu, Z. Shao, I. Gutman, and A. Klobučar, "Total domination and open packing in some chemical graphs," Journal of Mathematical Chemistry, vol. 56, no. 5, pp. 14811492, 2018.

[6] J.-B. Liu, X.-F. Pan, F.-T. Hu, and F.-F. Hu, "Asymptotic Laplacian-energy-like invariant of lattices," Applied Mathematics and Computation, vol. 253, pp. 205-214, 2015.

[7] J.-B. Liu and X.-F. Pan, "Minimizing Kirchhoff index among graphs with a given vertex bipartiteness," Applied Mathematics and Computation, vol. 291, pp. 84-88, 2016.

[8] J.-B. Liu, C. Wang, S. Wang, and B. Wei, "Zagreb indices and multiplicative zagreb indices of eulerian graphs," Bulletin of the Malaysian Mathematical Sciences Society, vol. 42, no. 1, pp. 67-78, 2019.

[9] J. B. Liu, J. Zhao, and Z. Zhu, "On the number of spanning trees and normalized Laplacian of linear octagonal-quadrilateral networks," International Journal of Quantum Chemistry, vol. 119, no. 17, Article ID e25971, 2019.

[10] Z. Shao, P. Wu, X. Zhang, D. Dimitrov, and J.-B. Liu, “On the maximum $\mathrm{ABC}$ index of graphs with prescribed size and without pendent vertices," IEEE Access, vol. 6, pp. 2760427616, 2018.

[11] Z. Shao, P. Wu, Y. Gao, I. Gutman, and X. Zhang, "On the maximum ABC index of graphs without pendent vertices," Applied Mathematics and Computation, vol. 315, pp. 298-312, 2017.
[12] Z. Cui and B. Liu, "On Harary matrix, Harary index and Harary energy," Match Communications in Mathematical and in Computer Chemistry, vol. 68, pp. 815-823, 2012.

[13] G.-X. Cai, M.-L. Ye, G.-D. Yu, and L.-F. Ren, "Hyper-Wiener index and Hamiltonicity of graphs," Ars Combinatoria, vol. 139, pp. 175-184, 2018.

[14] H.-B. Hua and M. Wang, "On Harary index and traceable graphs," Match Communications in Mathematical and in Computer Chemistry, vol. 70, pp. 297-300, 2013.

[15] H.-B. Hua and B. Ning, "Wiener index, Harary index and Hamiltonicity of graphs," Match Communications in Mathematical and in Computer Chemistry, vol. 78, no. 1, pp. 153-162, 2016.

[16] R. Liu, X. Du, and H. Jia, "Wiener index on traceable and Hamiltonian graphs," Bulletin of the Australian Mathematical Society, vol. 94, no. 3, pp. 362-372, 2016.

[17] R.-F. Liu, X. Du, and H.-C. Jia, "Some observations on Harary index and traceable graphs," Match Communications in Mathematical and in Computer Chemistry, vol. 77, no. 1, pp. 195-208, 2017.

[18] R. Li, "Wiener index and some Hamiltonian properties of graphs," International Journal of Mathematics and Soft Computing, vol. 5, no. 1, pp. 11-16, 2015.

[19] R. Li, "Harary index and some Hamiltonian properties of graphs," AKCE International Journal of Graphs and Combinatorics, vol. 12, no. 1, pp. 64-69, 2015.

[20] G.-D. Yu, L.-F. Ren, and X.-X. Li, "Wiener index, hyperwiener index, harary index and hamiltonicity properties of graphs," Applied Mathematics-A Journal of Chinese Universities, vol. 34, no. 2, pp. 162-172, 2019.

[21] T. Zeng, "Harary index and Hamiltonian property of graphs," Match Communications in Mathematical and in Computer Chemistry, vol. 70, pp. 645-649, 2013.

[22] B. Li and B. Ning, "Spectral analogues of Moon-Moser's theorem on Hamilton paths in bipartite graphs," Linear Algebra and Its Applications, vol. 515, pp. 180-195, 2017.

[23] B.-L. Li and B. Ning, "Spectral analogues of Erdos and MoonMoser's theorems on Hamilton cycles," Linear Multilinear Algebra, vol. 64, no. 11, pp. 1152-1169, 2016.

[24] G.-D. Yu, Y. Fang, and Y.-Z. Fan, "Spectral radius and hamiltonicity of graphs," Discussiones Mathematicae Graph Theory, vol. 39, pp. 951-974, 2019. 

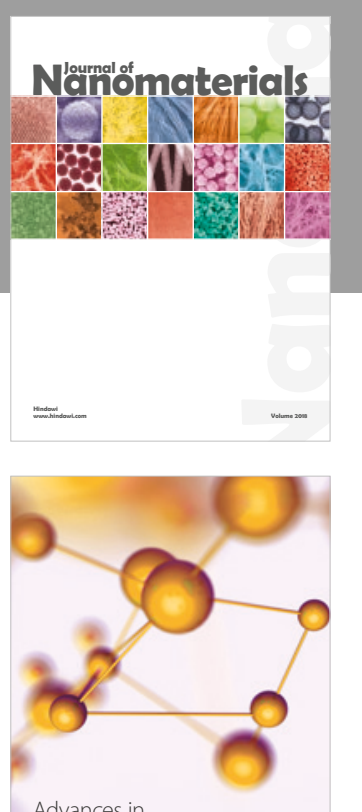

Physical Chemistry
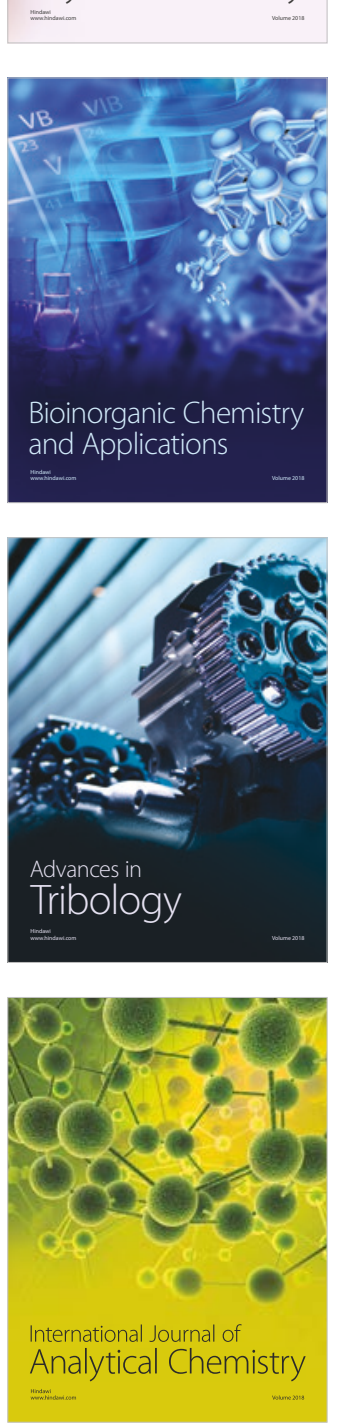

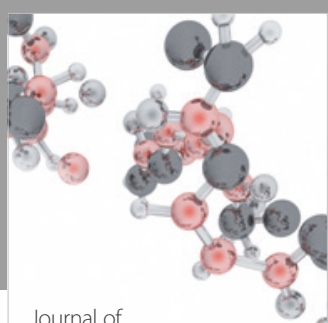

Analytical Methods

in Chemistry

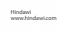

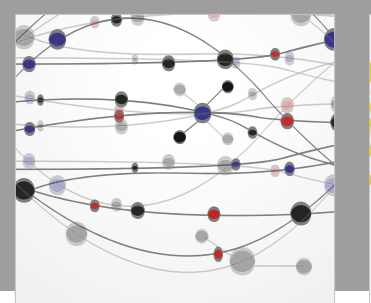

The Scientific World Journal

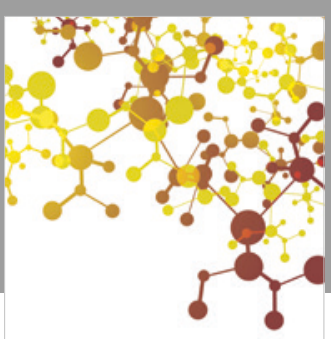

Journal of

Applied Chemistry
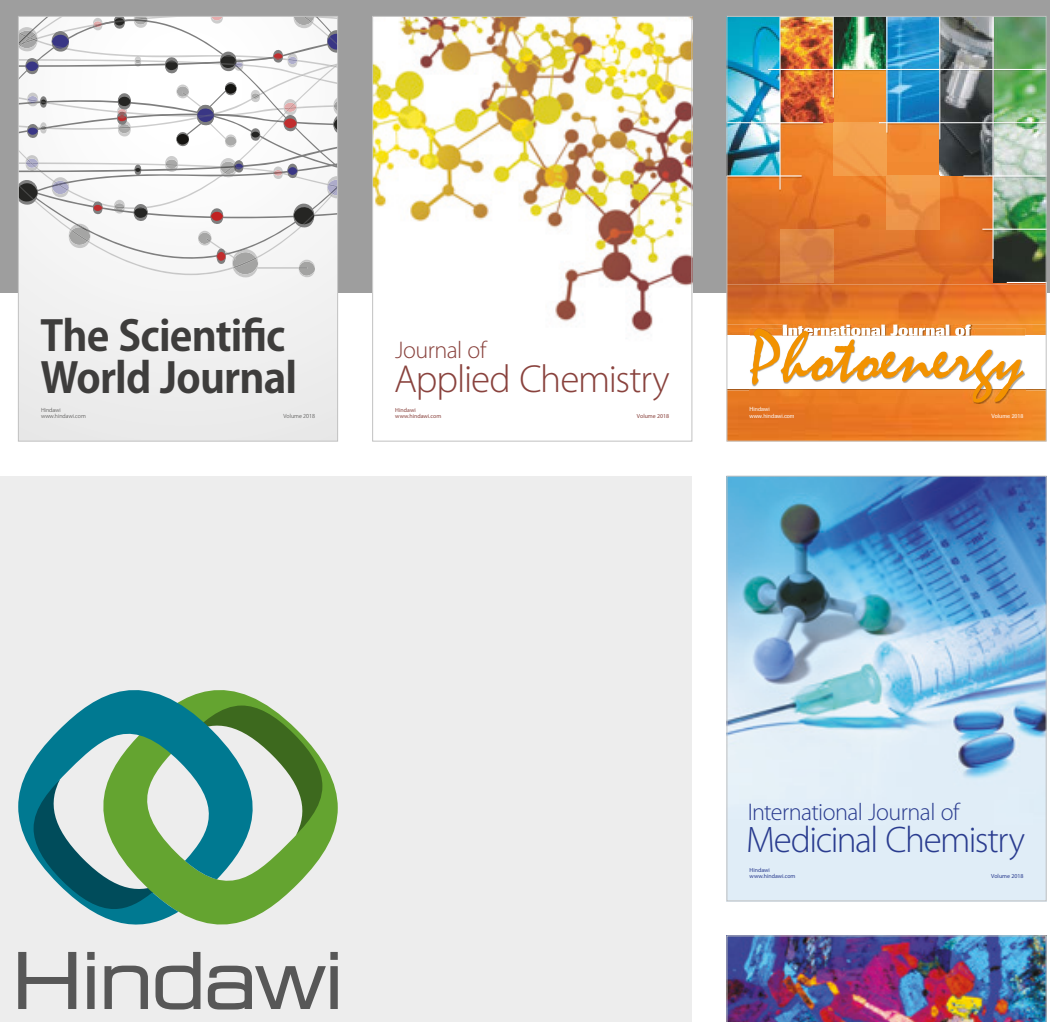

Submit your manuscripts at

www.hindawi.com
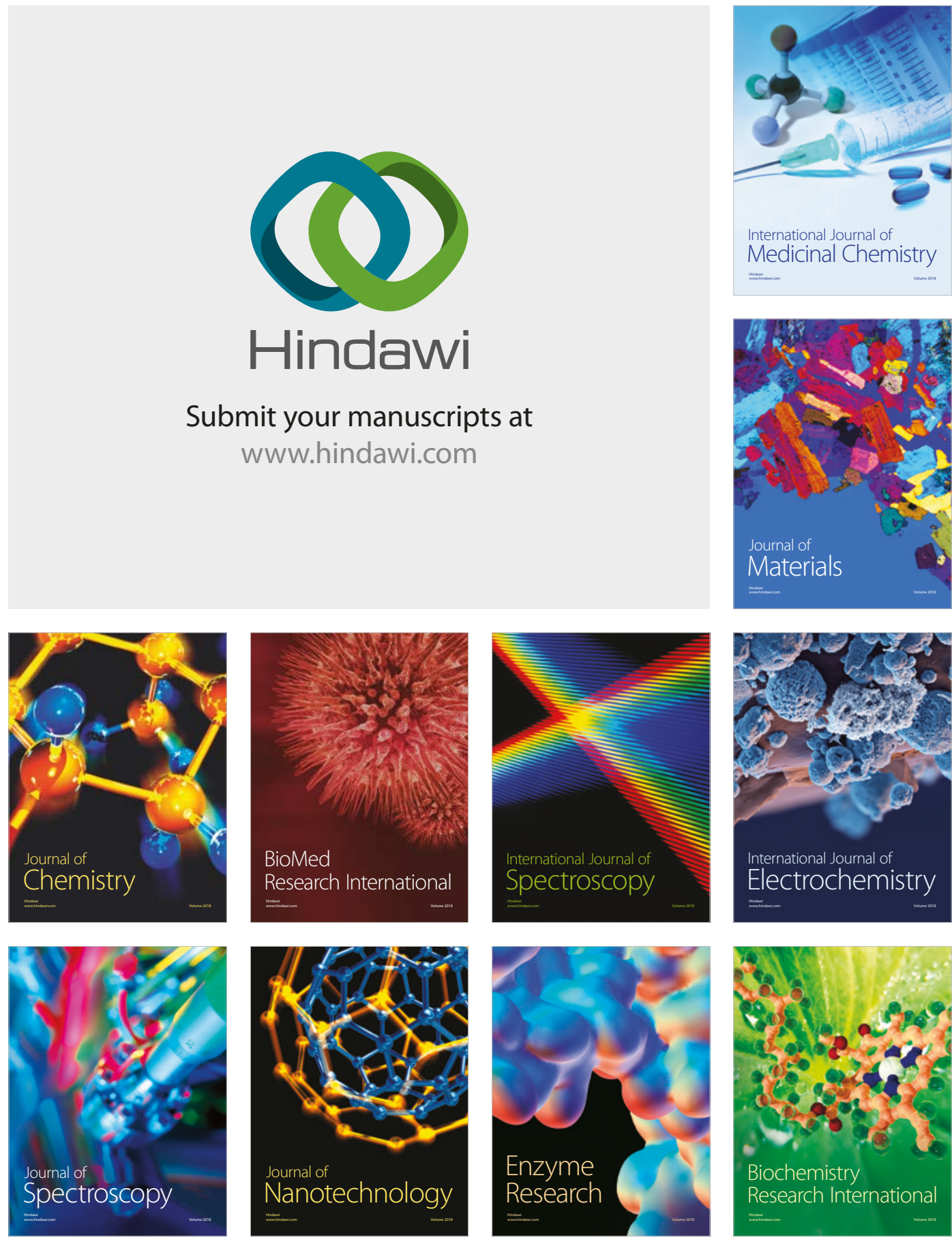
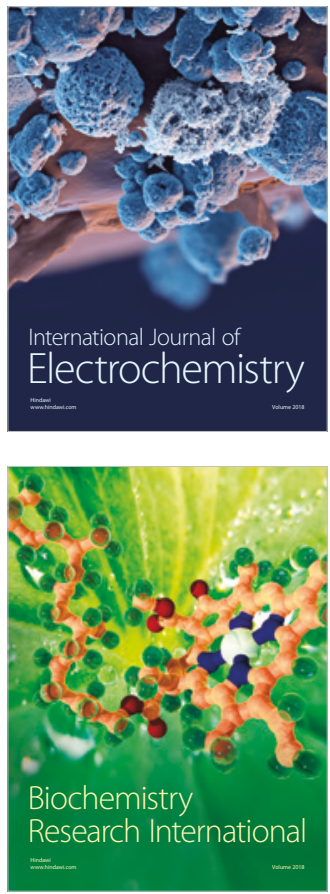